\title{
PacifiCorp Design Competition
}

\author{
Karen Kaminski, PacifiCorp 2010 Competition Chair
}

The problem statement for the 2010 PacifiCorp Design competition is out! We encourage students to consider participating on a team and faculty to support your students in getting involved. The first phase is due by May $1^{\text {st }}$, making this an excellent opportunity to incorporate the problem statement into your spring course work.

Teams of two students are typically from the same school, but members can work at a distance. If your proposal is accepted for phase 2, your assigned mentor will be from industry or another institution.

Additional information is available on the web site: http://aect.org/pacificorp/

\section{PacifiCorp Design Competition 2010 Problem}

A large public, urban hospital is trying to reduce costs as well as their carbon footprint. They would like all their employees to be "more green". Upper management has completed research to determine the prevailing culture of their competitors related to "green" initiatives, and they are convinced they have a compelling business case for going green. They want to drive this strategy down through the organization and realize that an initiative will be required to increase awareness and to shift employee attitudes. The hope is that this will lead to changes in employee behavior which will result in cost savings for the organization. In addition to enhancing their cost savings, they want to gain public recognition for their environmental consciousness and their work on environmental issues. The following information has guided their decision to move in this direction.

- Current advances in recycling capabilities,

- Data on environmental impact for use of alternative transportation, and

- Methods for conservation of natural resources.

They are looking to hire a team to develop/design/ provide training to create a sustainable change in employee attitude/behavior. This includes awareness of specific green initiatives leading to change in employee behavior at work and in their home environments.

\section{Within your plan:}

Specify the types of behaviors and associated attitudes to be changed. How would you address change management aspects of the initiative? What are strategies for increasing transfer of learning? Finally, identify methods for evaluating the impact of the intended changes on the workplace (e.g., reduced waste, increased productivity, electricity savings, etc.).

\section{First Phase of the Competition}

One subset of the hospital management has been charged with finding a team to create the training outlined above. This group would like your team to put together a document outlining your approach to creating the training they seek. The document should be detailed enough to give the group a sense of what you intend to do, but it should not be the final product in all its detail. It should be a discussion document for helping them decide whether to go forward with your team or not. As you begin this project, the creation of this document is your first task. This document must be submitted by May 1, 2010.

A client representative, Marcus Childress is available to answer your questions about the context/circumstances of the problem. You can reach him at mchildre@emporia.edu or 620-341-5677.

Up to six proposals from Phase One will be selected to proceed to Phase Two.

\section{Second Phase of the Competition}

Once there is agreement that your proposal represents a viable solution for the hospital, you will work with an independent learning consultant who will be assigned to you (a mentor) on what will become the final recommendation to the hospital. What you create will be a detailed description of the content you propose to include and the means by which you will deliver it. This will need to be explained to the hospital management in terms that they will understand. As much as possible, justifications for the decisions that went into your proposal will need to be provided. The creation of the documents that will be distributed to the hospital management is your second task. These documents are due on August 15, 2010. The top three proposals from Phase Two will enter the third and final phase.

\section{Third Phase of the Competition}

You've been part of the team that has put together all of the materials about how the hospital can successfully implement the green initiative they desire. Your liaison has now asked you to make a half-hour presentation to explain and distribute these materials to the hospital director and the rest of the upper management. Present your case for your solution.

Good luck! 